\title{
Inversionistificación en América Latina: problematización del mercado de arriendo para el caso chileno
}

\author{
Investification in Latin America: problematising \\ the letting market in the Chilean case
}

\author{
José-Francisco Vergara-Perucich y Carlos Aguirre Nuñez ${ }^{2}$
}

Fecha de recepción: 03-04-2019 - Fecha de aceptación: 04-07-2019

Hábitat y Sociedad (ISSN 2173-125X), n. . 12, noviembre de 2019, pp. 11-27.

http://dx.doi.org/10.12795/HabitatySociedad.2019.i12.02

\begin{abstract}
The article introduces how investification serve as an analytical framework for unpinning the increase of the buy-to-let phenomenon in a reduced letting market that produces a rise in letting prices. The Chilean neoliberal context and the dis-regulated condition of property-rent markets provides a chance for reviewing the insights of how housing and letting are undermining the affordability in the Latin American region. The first part is centred on an explorative statistical analysis based on CASEN survey 2009-2017 to identify the variations in the household whose incomes come from letting urban properties. To deepen the analysis, the second part explores the financial profitability of letting business in a specific borough of Santiago. This analysis employs two financial methods: the Yield and the Net Present Value (NPV). The results indicate that there is a higher concentration of this type of business in the wealthiest $10 \%$ while the profitability of letting business is high. These results demonstrate the existence of investificacion in Santiago and some public policy alternatives are offered to engage in a broader international discussion.
\end{abstract}

\section{Key words}

Letting; Housing; Rentism; Neoliberalism

\section{Resumen}

Se introduce el fenómeno de la inversionistificación como marco de análisis crítico para desentrañar las problemáticas asociadas al aumento de propietarios en un mercado de arriendos reducidos como parte de factores que inciden en el aumento de precio de arriendos. El contexto neoliberal chileno con un mercado de arriendos desregulados ofrece interesantes alcances al problema de la vivienda y el arriendo en ciudades de la región. El método se divide en dos partes. A nivel nacional se ha utilizado un método estadístico descriptivo a partir de la encuesta CASEN entre 2009 y 2017 para detectar cuánto ha variado la cantidad de hogares que perciben ingresos por arriendo de propiedades urbanas en este periodo. Para profundizar el análisis, se selecciona un barrio con heterogeneidad en valores de arriendo para estudiarlo mediante técnicas de evaluación de rentabilidad financiera (Yield y VAN). Los resultados de este estudio indican que ha aumentado la concentración de ingresos por arriendo de propiedades urbanas en el 10\% más rico y que en el barrio estudiado para todos los casos se observa una rentabilidad financiera alta. Con esto se demuestra la existencia de inversionistificación en Santiago y se ofrecen algunas alternativas de política pública.

Palabras clave

Arriendo; Vivienda; Rentismo; Neoliberalismo

1 Doctor e investigador de la Facultad de Arquitectura, Diseño y Construcción. Director del Centro Producción del Espacio de la Universidad de Las Américas, Santiago, Chile. E-mail: franciscovergarap@gmail.com

2 Director de la Escuela de Construcción de la Universidad de las Américas, Santiago, Chile. E-mail: caguirre@udla.cl 


\section{Introducción}

El incremento en las inversiones para arriendo de hogares es un fenómeno escasamente cubierto por la literatura existente sobre segmentos desaventajados. Hemos acuñado el término 'investification' para capturar el proceso de cómo los propietarios de vivienda la usan para alquilarla, con especial énfasis en el creciente rol del arriendo en el mercado de la vivienda privada y en detectar patrones espaciales de inversión para alquilar y así contribuir a las geografías de los desplazados en contextos urbanos contemporáneos (Hulse y Reynolds, 2018, p. 14). ${ }^{3}$

En 2018, Kath Hulse y Margaret Reynolds introducen el concepto ‘investification' para enmarcar los procesos en que un propietario utiliza la vivienda en alquiler bajo un esquema rentista. El marco analítico entregado por Hulse y Reynolds es aplicado a un estudio de caso en Sídney, Australia, para develar cómo el arriendo se ha convertido en un modelo de negocios para extraer renta desde la vivienda cuyos destinatarios son hogares de clase menos aventajada. En busca de explorar la aplicabilidad de este nuevo marco de análisis al contexto latinoamericano se ha realizado un estudio específico en Chile, en el barrio Santa Isabel de la comuna de Santiago, en busca de identificar si en este caso existe un proceso de 'investification'. Esta exploración permite revisar cómo este fenómeno produce exclusiones socioeconómicas, cuáles son las potenciales motivaciones de quienes aplican esta estrategia y qué potenciales implicaciones podría tener este proceso en el desarrollo urbano y en el diseño de nuevas políticas públicas que incorporen esta problemática a la agenda de ciudad.

El caso de la vivienda en Chile es frecuentemente usado para ilustrar cómo el modelo neoliberal puede dar forma a prácticas socio-espaciales orientadas a la extracción de valor desde los procesos urbanos (Boano y Vergara-Perucich, 2017). Ya sea mediante estudios de políticas públicas (Cociña, 2016), segregación (Ruiz-Tagle y López M., 2014; Sabatini, Rasse, Cáceres, Robles y Trebilcock, 2017), gentrificación (Lopez-Morales, Arriagada-Luco y Gasic-Klett y Meza-Corvalan, 2015; Inzulza-Contardo, 2016), financiarización (Gasic, 2018), verticalización (Vergara Vidal, 2017) y colusión (Vargas, 2016); el caso chileno cuenta con importantes aportes a discutir en un marco internacional sobre los efectos del modelo neoliberal en la vida urbana, por lo que la aplicación del nuevo método de estudio sobre 'investification' se constituye como una contribución metodológica útil para entender la dinámica de la ciudad como fuente de lucro.

En este artículo se ha traducido el término 'investification' como inversionistificación, proveniente de una conceptualización sobre cómo los inversionistas urbanos ('investors' en inglés) profitan del arriendo de propiedades urbanas, fijando el análisis en el estudio del comportamiento de los individuos (propietarios en relación con los arrendatarios), en vez de observar los macro-flujos de capital sobre estos procesos. Así, entonces, el artículo desarrolla un análisis microeconómico. No obstante, para otras investigaciones futuras, una aproximación macroeconómica podría ofrecer nuevos alcances metodológicos y otro tipo de reflexiones. El artículo parte presentando la inversionistificación en un marco de literatura más amplio sobre fenómenos de la vivienda en la ciudad neoliberal, seguido por una breve contextualización del régimen de propiedad privada y mercado del arriendo en Chile. Se sigue con la presentación de los métodos usados. Para realizar el estudio empírico se han realizado 
dos aproximaciones en dos escalas diferentes: a nivel nacional se ha utilizado un método estadístico descriptivo a partir de la encuesta CASEN entre 2009 y 2017 para detectar cuánto ha variado la cantidad de hogares que perciben ingresos por arriendo de propiedades urbanas en este periodo. Una segunda aproximación es barrial. Se ha seleccionado un barrio de la ciudad de Santiago que presente importantes variaciones en tipologías de departamentos con heterogeneidad en valores de arriendo para estudiarlo mediante dos técnicas de evaluación de rentabilidad financiera: Yield y Valor Actual Neto. A modo de breve anticipo de los resultados de este estudio, a nivel nacional ha aumentado la concentración de ingresos por arriendo de propiedades urbanas capturado por el 10\% más rico de los hogares, mientras que en caso particular del barrio Santa Isabel de Santiago Centro, se observa una rentabilidad financiera alta y una exclusión de la clase media en el acceso a estas viviendas en razón de los altos valores de arriendo. El artículo concluye que sí existe un proceso de inversionistificación en Santiago y se ofrecen algunas alternativas de política pública para instalar una reflexión sobre cómo reducir la exclusión por estas causas, con énfasis en qué relación tiene este fenómeno con la crisis del cuidado y la frágil seguridad social en contextos neoliberales como el chileno.

\section{La 'investification' como marco de análisis}

Para Henri Lefebvre, detrás de los aspectos más elementales de la vida cotidiana se esconden las verdaderas perversiones y contradicciones que caracterizan la sociedad capitalista, en situaciones concebidas teatralmente para generar escenas de vida que están cuidadosamente pensadas para extraer valor desde las relaciones sociales (Lefebvre, 1991). Dentro de los componentes emocionales más relevantes para configurar sensaciones de seguridad social en la vida cotidiana está el acceso a la vivienda (Marcuse en Brenner, Marcuse y Mayer, 2012). Cuando sistemas sociales tales como el neoliberalismo establecen barreras de acceso a la vivienda, se podría comenzar a ver el fenómeno de la alienación hacia los otros, en que los vecinos y miembros de una misma comunidad comienzan a competir entre sí para obtener posiciones seguras en la escala social, que reduzcan su vulnerabilidad, con lo que las personas se aíslan y se dividen temiendo por la hostilidad de otros miembros de la sociedad (Swain, 2012). Estas divisiones y aislaciones a causa de un modelo económico basado en la competencia entre individuos, se representan en disparidades urbanas materialmente visibles en la vivienda (Gilbert, 2013). En este contexto, el acceso a la propiedad privada es uno de los principales aspectos tendientes para generar seguridad ante una vida cotidiana amenazada por la riesgosa escenografía financiera neoliberal.

En el presente, el éxito de una ciudad tiende a medirse por la rentabilidad de sus inversiones urbanas (Pagano y Bowman, 1997). En la actualidad, gran parte de dichas inversiones se realiza con capital financiero, el cual en materia inmobiliaria se presenta como una inversión segura para quienes ostentan importantes niveles de riqueza, pero altamente riesgosa para quienes poseen poco capital de inversión o dependencia laboral (Durand, 2017).

$\mathrm{Al}$ implementarse el financiamiento inmobiliario para la compra de la casa propia, la expansión del mercado global de capitales se apoyó en el 
endeudamiento privado, estableciendo un vínculo íntimo entre la vida biológica de los individuos y el proceso global de extracción de renta y especulación (Rolnik, 2017, p. 37).

El acceso a la seguridad de la tenencia de vivienda concentrada en un grupo selecto de la población y el tratamiento de la vivienda como un activo financiero ha creado una situación que Raquel Rolnik (2017) ha descrito como la crisis global de inseguridad de tenencia. En esta crisis, los arrendatarios se encuentran altamente expuestos a las variaciones del mercado inmobiliario y a la especulación de algunos propietarios, que ven en la vivienda una inversión, asumiendo su valor de cambio y pormenorizando su valor de uso, generando así modelos de exclusión.

El proceso de exclusión de habitantes de un barrio por parte de nuevos propietarios o inversores se conoce tradicionalmente como gentrificación (Lees, Slater y Wyly, 2008). Este fenómeno cuenta con importantes avances y estudios en ciudades de América Latina (Hidalgo Dattwyler, 2014; Inzulza y Galleguillos, 2014; López-Morales, Gasic Klet y Meza Corvalán, 2014; Olivera, 2014; Sabatini et al., 2017). No obstante, el tópico del arriendo en la formación de mecanismos de exclusión/dominación en ciudades a partir de la relación arrendatario/ propietario cuentan con una nueva metodología de aproximación que fue presentada como investification y que hemos traducido como inversionistificación. Kath Hulse y Margaret Reynolds (2018) plantean el concepto de inversionistificación para explicar un proceso en el que una aumentada capacidad de compra por parte de un inversor le permite adquirir propiedades en barrios de menores ingresos, acelerando el incremento del valor de dichas propiedades y arriendos en el sector en desmedro de los sectores económicamente menos aventajados que lo habitan, para así extraer renta de esos procesos. Para Hulse y Reynolds (ob. cit.), la adquisición de vivienda por parte del inversor tiende a realizarse para luego arrendar las propiedades en el mercado de la vivienda local y así paliar la vulnerabilidad financiera y generar un instrumento privado de seguridad social futura ante la fragilidad de sistemas de salud y pensiones. En este estudio, las autoras plantean que los propietarios no son necesariamente ricos, sino que han sido orientados o bien conocen el sistema crediticio para acceder a estos mecanismos de generar rentabilidad en busca de asegurar un futuro incierto, más que por un ánimo de avaricia. No obstante, el modelo bien puede utilizarse para instalar una máquina extractiva de valor a partir del arriendo que el inversionista Felipe Yaluff ha denominado como vacas lecheras que son "propiedades que se arrienden sin vacancia y aumenten su valor como activos a través del tiempo" y "se caracteriza por obtener una excelente rentabilidad, porque se trata de un bien raíz muy cotizado" (Yaluff Portilla, 2016, p. 70). La inversionistificación es un modelo de negocios que merece una mirada con mayor detención en las metrópolis chilenas dado que podrían ser indicativas de procesos de exclusión de clases vulnerables que se originan en la incapacidad de acceder a arriendos en viviendas con buena localización.

Según Hulse y Reynolds (2018), los arrendatarios de estas propiedades tienden a ser hogares de menores ingresos con incapacidad de adquirir vivienda, que, mediante la necesidad básica del habitar en tanto valor de uso, activan el ciclo de ganancias que favorece a los inversores a partir de la vivienda en tanto valor de cambio. Esto se inscribe dentro de un fenómeno que es reciente en algunos países europeos y anglo- 
sajones, al menos en su versión más masiva, conocida como buy-to-let, o comprar para arrendar. Entonces, el aporte metodológico de la inversionistificación es revisar cómo los hogares de mayores ingresos invierten en la compra de propiedades en sectores donde no les interesa vivir sino rentar y qué efectos puede producir este fenómeno en los hogares de menores ingresos. La principal pregunta que se plantea esta línea investigativa desarrollada por Hulse y Reynolds (ob. cit.) apunta a entender las razones que hay detrás de estas decisiones para así aprender cuáles son los patrones que se dan entre quienes operan bajo este esquema de rentas urbanas, buscando saber si este fenómeno de microacumulación puede ser gestionado mediante políticas públicas.

Así, la inversionistificación articula la teoría de la financialización de la vivienda con la gentrificación, a la vez que entrega potenciales insumos para investigaciones referidas a la especulación económica con la vivienda como activo financiero (Morales Soler, Alonso Mallén y Moreno Cruz, 2017), a la vez que presenta un nuevo enfoque a potenciales causas de la segregación residencial (Leal, 2012), otorgando una mirada más material y espacial a un análisis que podría esfumarse en la abstracción propia de las relaciones financieras, contribuyendo así a complementar los problemas ausentes de la Nueva Agenda Urbana, la cual carecía de posturas más rotundas a situaciones urbanas críticas producidas como consecuencia de la neoliberalización de los procesos urbanos (Rodríguez y Sugranyes, 2017). También, la inversionistificación pone el acento en el inversor, generando una figura de poder más definida que otras abstracciones conceptuales como hablar de la neoliberalización del desarrollo inmobiliario o del mercado de la vivienda. Siendo un concepto nuevo, nos interesa revisar cómo se entiende este fenómeno en el contexto chileno.

\section{Propiedad, arriendo y mercado inmobiliario en Chile}

El acceso a la propiedad de vivienda es una de las principales prioridades de los chilenos (Landon, 2017), consecuencia de una larga tradición nacional en que el Estado ha trabajado en procesos subsidiarios y legislativos, para facilitar el acceso a la tenencia de vivienda de los Chilenos (Navarrete y Navarrete, 2016). No obstante, el fomento de una política habitacional en que el Estado apoya con fondos para mercados de vivienda en busca de aumentar el número de propietarios, podría generar efectos tales como localización de vivienda en periferias o reducción de mercado de arriendos, reduciendo así la movilidad social y potencialmente generando una perpetuación de la desigualdad en el acceso a bienes públicos (OECD, 2013). Esta mirada se confirma con los datos, dado que en la actualidad el $63,7 \%$ de los hogares es propietario de una vivienda y solo un $25 \%$ ocupa una vivienda en situación de arrendatario (MIDESO, Ministerio de Desarrollo Social, 2017). Ante la evidencia reciente y la experiencia de la crisis inmobiliaria de 2008, expertos plantean que la tendencia de ampliar la tenencia de vivienda privada debiese desarrollarse bajo ciertos resguardos (Harvey, 2015) dado que gran parte de dicho acceso a la propiedad se desarrolla mediante endeudamiento con entidades financieras, algo particularmente visible en el caso chileno donde el $62 \%$ de las propiedades se han comprado sobre la base de créditos hipotecarios (MIDESO, 2018). 
En 2002, Jean Cummings y Denise DiPasquale (en Glaeser y Meyer, 2002) planteaban que la política de vivienda en Chile contribuye a distorsiones espaciales que, entre otras consecuencias, produce una limitación para acceder al mercado formal del arriendo en el que pueden participar con mayor libertad los hogares más ricos. La evidencia reciente indica que en ciudades como Santiago existe un alto nivel de exclusión por el precio de la vivienda generada por las políticas urbanas que regulan los mercados de suelo, donde la aparición de nuevos edificios en altura se acompaña por la expulsión de los residentes antiguos de los barrios (López-Morales, 2016, en Boano y Vergara-Perucich, 2017). La relación entre la verticalización de la ciudad y su crecimiento tiene que ver con tasas de urbanización — que en Chile se acerca al $90 \%$ de personas vivienda en centros urbanos-, donde la capitalización se refleja en vivienda en altura que resulta altamente regresiva para la sociedad dado que exige que ciertos actores con capital suficiente para verticalizar comiencen a concentrar la inversión en áreas seguras para la inversión, privilegiando sectores consolidados o centrales y excluyendo al resto de los espacios de la ciudad, en un proceso de producción urbana caracterizada como especulación financiera desregulada (Graham, 2016). Santiago es un buen ejemplo en América Latina de este proceso de verticalización (Vergara Vidal, 2017). A su vez, la concentración de la inversión y formación de proyectos inmobiliarios se beneficia en procesos de alta demanda de vivienda (situación constante en Chile), bajo un fenómeno en que el precio de la vivienda se fija por la escasa necesidad de aumentar la competitividad del mercado, generando una colusión tácita entre actores (Vargas, 2016), cuya principal meta es la renta y no la provisión de vivienda. De esta colusión tácita, se producen precios de vivienda que crecen a un ritmo propio. Esto impacta también los mercados de arriendo.

Para Ernesto López-Morales y Nicolás Herrera (2018), la evidencia reciente es rotunda en relación a la necesidad de problematizar el mercado de arriendos en Chile. Entre 2009 y 2015, el precio promedio del arriendo experimentó un aumento de $31,2 \%$, a la vez que se redujo el tamaño de las viviendas medidas en $\mathrm{m}^{2}$ por unidad. A pesar de aquello, la vivienda de tamaños inferiores a $30 \mathrm{~m}^{2}$ aumentó en un $133 \%$ en cuanto a su disponibilidad de venta en el mercado. Para el mismo periodo, mientras en 2009 un 18\% de los hogares eran arrendatarios, ya en 2017 esa cifra llega al 25\% de los hogares (ob. cit.). Debido a que la vivienda es el componente que más impacta en los presupuestos de los hogares, el estudio de fenómenos como la inversionistificación en zonas urbanas puede iluminar la existencia de nuevos mecanismos de extracción de valor desde la cotidianeidad de los hogares en Chile.

Según resultados recientes, en Chile los hogares destinan un 56\% del ingreso mensual al pago de arriendo en vivienda, transporte público y alimentación diaria, pero al segmentar por deciles socioeconómicos se observa que mientras los hogares más pobres destinan un 118\% de sus ingresos al pago de gastos fijos, es decir, la vida urbana formal se les hace insostenible financieramente, los hogares más ricos cubren sus necesidades básicas con un $18 \%$ del ingreso mensual por hogar (Vergara Perucich, 2018) transporte público y canasta básica alimentaria de las principales ciudades de Chile, mediante un análisis de datos obtenidos a partir de la encuesta CASEN 2015, el Instituto Nacional de Estadísticas y la Subsecretaría de Transportes (SECTRA. En este contexto, profundizaremos en cómo los arriendos y la inversionistificación inciden en los hogares. 


\section{Método}

Para investigar el fenómeno de la inversionistificación en Chile desarrollamos dos aproximaciones escalares. La primera es a nivel nacional para determinar la magnitud de hogares que utilizan el arriendo de otras propiedades urbanas para rentar. Una segunda aproximación se basó en el estudio de un barrio en particular en donde una presencia de edificios a la venta se habían capitalizado recientemente (desde hace 10 o 15 años), privilegiando así la existencia de departamentos que ya habían estado un tiempo dentro de lo que podríamos llamar como marco de inversionistificación (investification framework). En primera instancia, utilizamos los datos provistos por la Encuesta de Caracterización Socioeconómica de Chile (CASEN). Esta encuesta se realiza cada dos a tres años para determinar las variaciones en un amplio espectro de factores de estudio que permiten construir perfiles sociales y determinar focos específicos donde deben actuar las políticas públicas. Como parte del muestreo se hacen preguntas referidas a la vivienda y a los ingresos. Entre las preguntas por ingresos existe una pregunta que indaga si las personas han percibido ingresos por arriendos de propiedades urbanas, al igual que se hace una pregunta para identificar a qué monto ascienden dichos ingresos. Así, también, la encuesta permite identificar las comunas donde viven personas que más concentran ingresos por concepto de arriendo de propiedades y las comunas donde existe una mayor concentración de arrendatarios. En este sentido, la encuesta permite espacializar por comunas el fenómeno de la inversionistificación. Debido a que la encuesta se aplica cada cierto período, también su análisis permitiría identificar los aumentos del precio del arriendo. En este caso, estudiamos las variaciones entre los años 2009 y 2015. Hemos tenido que excluir el año 2017 (último año disponible) porque la pregunta sobre ingresos por arriendo de propiedades urbanas tuvo un cambio metodológico que impide su comparación con años anteriores.

La segunda aproximación al problema se enfoca en estudiar el barrio conocido como Santa Isabel. De una base de datos de 1180 propiedades hicimos un primer muestreo aleatorio de 120 para definir ciertos patrones comunes que fuesen representativos. Finalmente, exponemos los resultados de un análisis con 31 exponentes que resumen las características tipológicas, de valores comerciales y de localización, para representar las observaciones económicas. Para definir las ganancias obtenidas por propietarios en el proceso de arriendo de departamentos aplicamos una fórmula Yield que identifica el flujo de dinero obtenido por una inversión en un determinado período de tiempo (Duffie y Kan, 1996; Tsatsaronis y Zhu, 2004), para así anticipar qué tan rentable es el negocio de comprar para arrendar en el barrio Santa Isabel. El resultado del análisis Yield se complementa presentando el resultado de estudio del Valor Actual Neto - VAN- de la inversión (Hanafizadeh y Latif, 2011; Kolb, 2018) y de la renta estimada mensual por el arriendo de las tipologías estudiadas.

\section{Resultados}

En un estudio inicial de cómo el valor de arriendo ha ido aumentando en relación a los ingresos por hogar en Chile (Cuadro 1) se puede observar que el primer decil socioeconómico (de menores ingresos) ha expe- 
rimentado un aumento en la carga mensual para poder pagar un constante aumento en el peso del precio del arriendo sobre los presupuestos del hogar. Entre 2009 y 2017 el aumento del peso del arriendo sobre el promedio de los presupuestos de los hogares llegó a una variación de un $47,4 \%$. Por su parte el $10 \%$ más rico ha visto cómo la presión sobre el presupuesto de los hogares se mantiene bajo el $20 \%$ del ingreso, lo cual se puede considerar como moderado y el aumento entre 2009 y 2017 ha sido solo de un 2,13\%. El hecho que en 2017 el arriendo resulte impagable para el $10 \%$ de menores ingresos podría correlacionarse con el considerable número de 473517 hogares hacinados (Instituto Nacional de Estadísticas, 2018) y la cantidad de habitantes expulsados hacia alternativas de vivienda informal (Flores, 2017; Vergara-Perucich, 2017), llegando a un incremento de un 55,42\%. Estos datos, además, son indicativos de la capacidad de pago de los hogares para acceder a créditos hipotecarios. En general, en el sistema financiero chileno se establece que un 25\% del ingreso por hogar puede destinarse a deuda hipotecaria. Para el arriendo, en cambio, la oferta actual exige como límite un gasto máximo de un $20 \%$ del ingreso del hogar para destinar a vivienda. Bajo este parámetro, los datos de 2017 indican que los 6 primeros deciles no pueden acceder con facilidad al pago de arriendos.

\begin{tabular}{|c|c|c|c|c|c|c|}
\hline $\begin{array}{l}\text { Deciles } \\
\text { Socioeconómicos }\end{array}$ & 2009 & 2011 & 2013 & 2015 & 2017 & $\begin{array}{c}\text { Diferencia } \\
2017-2009\end{array}$ \\
\hline 1 & $47,35 \%$ & $49,52 \%$ & $48,67 \%$ & $52,86 \%$ & $94,75 \%$ & $47,40 \%$ \\
\hline 2 & $26,66 \%$ & $29,06 \%$ & $29,95 \%$ & $31,16 \%$ & $39,76 \%$ & $13,10 \%$ \\
\hline 3 & $22,51 \%$ & $23,94 \%$ & $25,55 \%$ & $25,91 \%$ & $31,92 \%$ & $9,41 \%$ \\
\hline 4 & $19,84 \%$ & $20,85 \%$ & $21,12 \%$ & $22,79 \%$ & $26,27 \%$ & $6,43 \%$ \\
\hline 5 & $17,20 \%$ & $17,64 \%$ & $19,56 \%$ & $19,56 \%$ & $22,81 \%$ & $5,61 \%$ \\
\hline 6 & $15,68 \%$ & $17,27 \%$ & $19,32 \%$ & $18,25 \%$ & $20,20 \%$ & $4,52 \%$ \\
\hline 7 & $14,86 \%$ & $15,33 \%$ & $16,12 \%$ & $16,60 \%$ & $17,84 \%$ & $2,98 \%$ \\
\hline 8 & $12,78 \%$ & $13,36 \%$ & $14,90 \%$ & $15,34 \%$ & $15,79 \%$ & $3,00 \%$ \\
\hline 9 & $11,50 \%$ & $11,50 \%$ & $13,71 \%$ & $13,93 \%$ & $13,98 \%$ & $2,48 \%$ \\
\hline 10 & $7,92 \%$ & $8,48 \%$ & $9,66 \%$ & $10,09 \%$ & $10,05 \%$ & $2,13 \%$ \\
\hline Promedios & $19,63 \%$ & $20,69 \%$ & $21,86 \%$ & $22,65 \%$ & $29,34 \%$ & \\
\hline
\end{tabular}

Cuadro 1. Relación valor de arriendo con ingreso monetario por hogar a nivel nacional. Fuente: Elaboración propia en base a los datos de la encuesta CASEN, 2006-2017
Esto se conecta con la teoría de la inversionistificación ante un notorio aumento de la concentración de hogares que perciben ingresos por arriendo de propiedades urbanas en los dos deciles de mayores ingresos (Cuadro 2). Según los datos recopilados, la concentración de este tipo de negocios en el $10 \%$ más rico ha presentado una variación del 22,98\% para 2015, año en el que ya se podía revisar en el Cuadro 2 que $62,94 \%$ de este tipo de operaciones comerciales se concentraba en los 2 deciles de más altos ingresos. Estos antecedentes permiten identificar que el fenómeno de la inversionistificación en Chile sí estaría presente, afectando el mercado de arriendo de la vivienda y en el cual los que se ven más estancados son los hogares pertenecientes a los deciles V, VI y VII, quienes no son sujetos de subsidio estatal ni tampoco cuentan con el capital suficiente para acceder a propiedad de vivienda.

Los deciles del V al VIII se ven cada vez más presionados por el esfuerzo del hogar para pagar el arriendo, el cual ha ido en aumento, 
mientras no existen mayores variaciones en los IX y X. Sin embargo, la concentración de ingreso producto de arriendo de propiedades urbanas aumenta significativamente, lo que permite levantar la hipótesis de que los deciles IX y X han aumentado su adquisición de vivienda en relación a periodos anteriores y que sus potenciales arrendatarios estarían en los deciles del V al VIII. Ante la aparente existencia de un fenómeno de inversionistificación, se realizará un estudio específico caracterizado por su dinamismo inmobiliario en los últimos 15 años. Este sector es conocido como Santa Isabel, se encuentra en la comuna de Santiago Centro, que es el área de la ciudad que ha experimentado el mayor número de metros cuadrados construidos en una alta concentración de nuevos propietarios (López-Morales, 2016).

\begin{tabular}{|c|c|c|c|c|c|}
\hline $\begin{array}{l}\text { Decil autónomo } \\
\text { regional }\end{array}$ & 2009 & 2011 & 2013 & 2015 & Variación \\
\hline I & $8,01 \%$ & $8,48 \%$ & $8,92 \%$ & $8,39 \%$ & $4,72 \%$ \\
\hline II & $7,51 \%$ & $8,99 \%$ & $10,25 \%$ & $8,47 \%$ & $12,87 \%$ \\
\hline III & $8,98 \%$ & $7,81 \%$ & $11,10 \%$ & $10,05 \%$ & $11,93 \%$ \\
\hline IV & $8,78 \%$ & $8,43 \%$ & $11,91 \%$ & $9,86 \%$ & $12,39 \%$ \\
\hline V & $9,90 \%$ & $10,29 \%$ & $13,04 \%$ & $10,87 \%$ & $9,87 \%$ \\
\hline VI & $11,71 \%$ & $11,33 \%$ & $15,02 \%$ & $12,57 \%$ & $7,30 \%$ \\
\hline VII & $13,62 \%$ & $12,09 \%$ & $15,86 \%$ & $14,79 \%$ & $8,54 \%$ \\
\hline VIII & $18,45 \%$ & $16,45 \%$ & $19,77 \%$ & $19,26 \%$ & $4,42 \%$ \\
\hline IX & $23,04 \%$ & $20,93 \%$ & $24,21 \%$ & $24,27 \%$ & $5,37 \%$ \\
\hline X & $31,45 \%$ & $33,32 \%$ & $36,06 \%$ & $38,67 \%$ & $22,98 \%$ \\
\hline
\end{tabular}

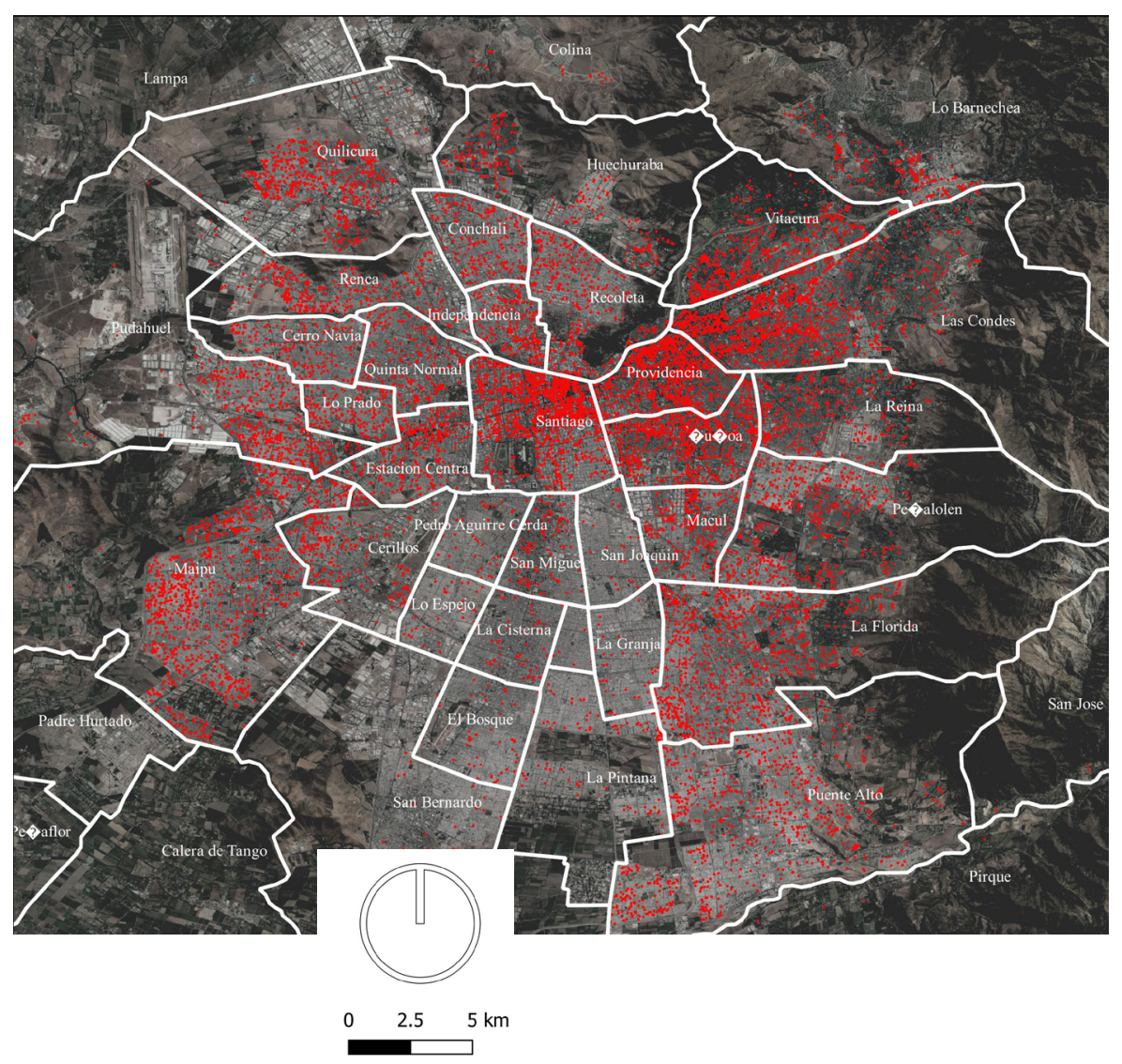

Cuadro 2. Hogares que perciben ingresos por arriendo de propiedades urbanas a nivel nacional. Fuente: Elaboración propia en base al Archivo Histórico de Encuesta CASEN del Ministerio de Desarrollo Social.

Figura 1. Mapa del Gran Santiago en el cual los puntos expresan los sectores donde existe mayor presencia de inversiones en 2 o más departamentos por una misma persona. Se observa especial concentración en el sector central de la comuna de Santiago, donde se aplica el estudio de caso. Fuente: Autores en base a $\operatorname{lnCiti.com.~}$ 


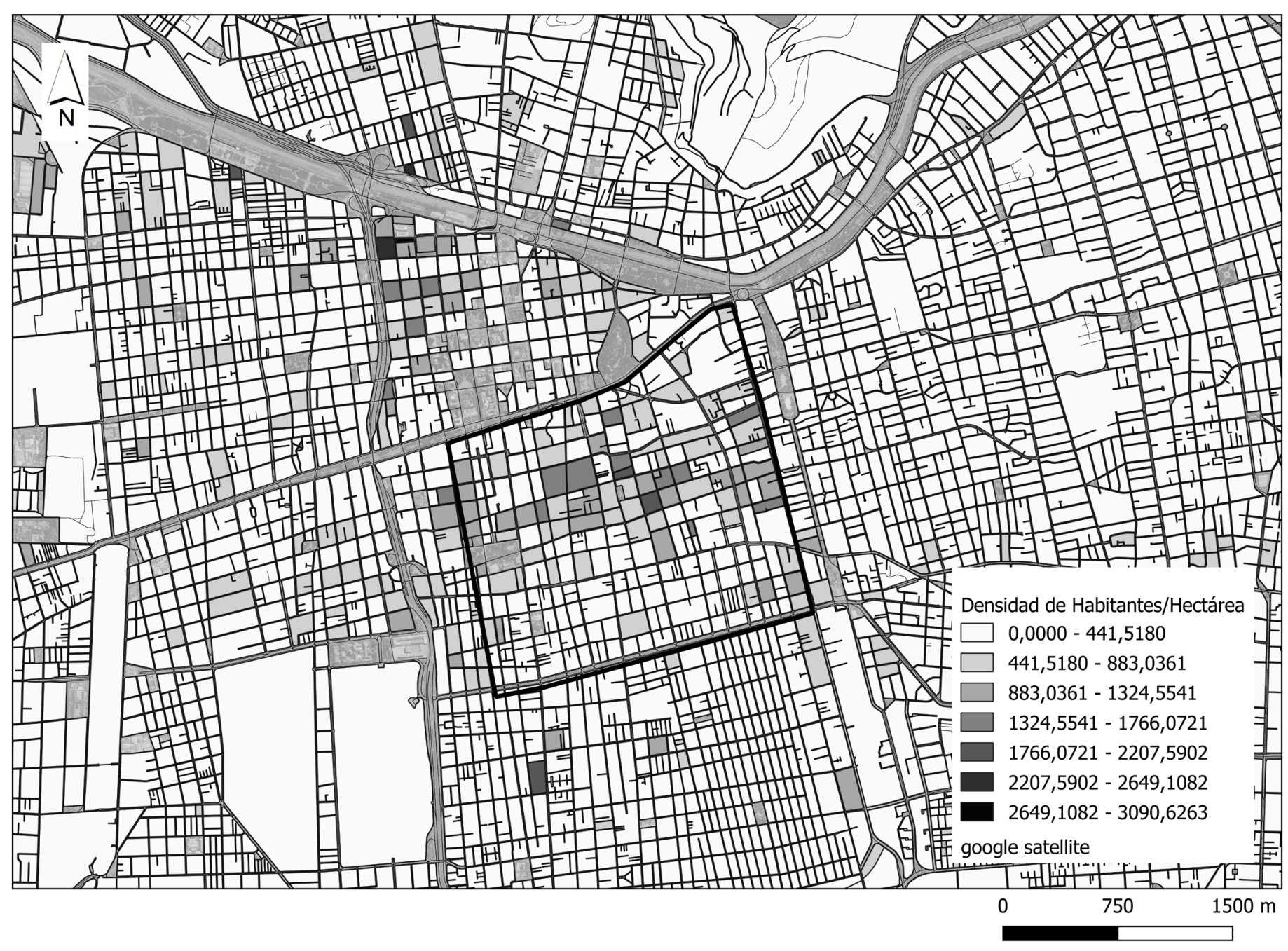

Figura 2. Mapa de densidad de habitantes por hectárea en sector Santiago Centro. El marco negro indica sector de análisis de este estudio conocido como barrio Santa Isabel. Fuente: Elaboración propia en base a los datos del CENSO 2017

4 En marzo de 2019, 1 UF= 42,3 USD. 5 En números romanos $\mathrm{I}=$ decil de menores ingresos, $\mathrm{X}=$ decil de más altos ingresos. Para el arriendo, las condiciones comerciales explícitas corresponden a $1 / 5$ del ingreso total del hogar. Es decir, para hacer las estimaciones se considera que un $20 \%$ del ingreso es imputable al alquiler.
Como primera aproximación, se desarrolla un levantamiento de los sectores de la ciudad donde un mismo propietario cuenta con dos o más departamentos, en el supuesto que esos departamentos residenciales excedentes son utilizados para el arriendo (Figura 1). Luego se hace un zoom al sector de estudio (Figura 2). Estos datos fueron recabados durante el mes de marzo de 2019. En este levantamiento se consideraron los precios de arriendo, precios de venta y algunos atributos de la vivienda (superficie, cantidad de habitaciones, condiciones comerciales). Los valores están expresados en pesos chilenos, en dólares americanos y en Unidades de Fomento (UF). La UF ${ }^{4}$ es un valor de cambio indexados sobre la base de las variaciones de la inflación ampliamente usado en Chile para la compra y venta de bienes inmuebles, permitiendo que su precio siempre tenga el mismo valor adquisitivo. Entonces, en el Cuadro 3 se registran los precios de arriendo de la muestra aplicada al barrio de Santa Isabel, junto con la indicación de aquellos grupos socioeconómicos divididos por decil que pueden acceder a arrendar las viviendas observadas a partir del ingreso promedio por hogar. ${ }^{5}$ La ubicación indica la calle referencia donde está el edificio de la unidad observada. 
Inversionistificación en América Latina: problematización del mercado de arriendo para el caso chileno

\begin{tabular}{|c|c|c|c|c|c|c|c|c|c|}
\hline $\begin{array}{l}\text { Precio de } \\
\text { Arriendo } \\
\text { (CLP) }\end{array}$ & $\begin{array}{l}\text { Precio de } \\
\text { Arriendo } \\
\text { (UF) }\end{array}$ & $\begin{array}{l}\text { Decil } \\
\text { que } \\
\text { puede } \\
\text { pagar }\end{array}$ & Ubicación & Yield & $\begin{array}{l}\text { Ingreso } \\
\text { para } \\
\text { Acceder a } \\
\text { Compra }\end{array}$ & $\begin{array}{l}\text { Utilidades } \\
\text { (UF) }\end{array}$ & VAN & $\begin{array}{l}\text { Plusavalía } \\
\text { a } 25 \text { años } \\
\text { (USD) }\end{array}$ & $\begin{array}{l}\text { Plusvalía } \\
\text { Anual } \\
\text { (USD) }\end{array}$ \\
\hline$\$ 350000$ & 12,73 & VIII & $\begin{array}{l}\text { Santa } \\
\text { Isabel }\end{array}$ & $8,66 \%$ & $\$ 829853$ & 1525 & 1846 & 77027,60 & 256,76 \\
\hline$\$ 430000$ & 15,64 & IX & $\begin{array}{l}\text { Santa } \\
\text { Isabel }\end{array}$ & $8,27 \%$ & $\$ 1048729$ & 1793 & 2270 & 94744,57 & 315,82 \\
\hline$\$ 240000$ & 8,73 & VII & Tarapacá & $9,97 \%$ & $\$ 501539$ & 1232 & 1265 & 52779,88 & 175,93 \\
\hline$\$ 340000$ & 12,36 & VIII & Jofré & $9,57 \%$ & $\$ 731910$ & 1686 & 1800 & 75104,81 & 250,35 \\
\hline$\$ 290000$ & 10,55 & VIII & $\begin{array}{l}\text { Sta } \\
\text { Victoria }\end{array}$ & $6,88 \%$ & $\$ 864799$ & 774 & 1508 & 62916,66 & 209,72 \\
\hline$\$ 400000$ & 14,55 & IX & Jofré & $7,66 \%$ & $\$ 1053327$ & 1453 & 2103 & 87742,33 & 292,47 \\
\hline$\$ 360000$ & 13,09 & VIII & Carmen & $11,22 \%$ & $\$ 662477$ & 2096 & 1918 & 80047,37 & 266,82 \\
\hline$\$ 350000$ & 12,73 & VIII & $\begin{array}{l}\text { V. } \\
\text { Mackenna }\end{array}$ & $8,68 \%$ & $\$ 828014$ & 1530 & 1846 & 77035,20 & 256,78 \\
\hline$\$ 305500$ & 11,11 & VIII & $\begin{array}{l}\text { Santa } \\
\text { Isabel }\end{array}$ & $12,66 \%$ & $\$ 502919$ & 1943 & 1630 & 68020,89 & 226,74 \\
\hline$\$ 400000$ & 14,55 & IX & E. Ramirez & $7,79 \%$ & $\$ 1034014$ & 1506 & 2105 & 87822,18 & 292,74 \\
\hline$\$ 320000$ & 11,64 & VIII & Marin & $10,27 \%$ & $\$ 644084$ & 1711 & 1697 & 70812,45 & 236,04 \\
\hline$\$ 380000$ & 13,82 & IX & Tarapaca & $8,74 \%$ & $\$ 879514$ & 1715 & 2008 & 83805,49 & 279,35 \\
\hline$\$ 407000$ & 14,8 & IX & $\begin{array}{l}\text { Santa } \\
\text { Isabel }\end{array}$ & $9,54 \%$ & $\$ 864799$ & 2050 & 2160 & 90151,23 & 300,5 \\
\hline$\$ 400000$ & 14,55 & IX & Curico & $6,90 \%$ & $\$ 1168283$ & 1135 & 2091 & 87267,03 & 290,89 \\
\hline$\$ 350000$ & 12,73 & VIII & San Isidro & $10,05 \%$ & $\$ 717656$ & 1835 & 1857 & 77491,49 & 258,3 \\
\hline$\$ 315500$ & 11,47 & VIII & $\begin{array}{l}\text { Santa } \\
\text { Isabel }\end{array}$ & $11,47 \%$ & $\$ 570513$ & 1865 & 1679 & 70069,16 & 233,56 \\
\hline$\$ 430000$ & 15,64 & IX & $\begin{array}{l}\text { Santa } \\
\text { Isabel }\end{array}$ & $8,34 \%$ & $\$ 1053327$ & 1780 & 2270 & 94725,55 & 315,75 \\
\hline$\$ 260000$ & 9,45 & VIII & Marin & $12,12 \%$ & $\$ 449119$ & 1595 & 1382 & 57652,10 & 192,17 \\
\hline$\$ 365000$ & 13,27 & VIII & Tarapaca & $9,63 \%$ & $\$ 754442$ & 1897 & 1937 & 80831,00 & 269,44 \\
\hline$\$ 500000$ & 18,18 & IX & Argomedo & $9,04 \%$ & $\$ 1122300$ & 2353 & 2654 & 110734,57 & 369,12 \\
\hline$\$ 230000$ & 8,36 & VII & $\begin{array}{l}\text { Sta } \\
\text { Victoria }\end{array}$ & $6,95 \%$ & $\$ 662477$ & 678 & 1193 & 49786,73 & 165,96 \\
\hline$\$ 290000$ & 10,55 & VIII & $\begin{array}{l}\text { Santa } \\
\text { Isabel }\end{array}$ & $9,89 \%$ & $\$ 607298$ & 1485 & 1533 & 63981,32 & 213,27 \\
\hline$\$ 440000$ & 16 & IX & $\begin{array}{l}\text { Santa } \\
\text { Isabel }\end{array}$ & $8,21 \%$ & $\$ 1067121$ & 1851 & 2324 & 96996,26 & 323,32 \\
\hline$\$ 410000$ & 14,91 & IX & $\begin{array}{l}\text { Santa } \\
\text { Isabel }\end{array}$ & $8,39 \%$ & $\$ 993550$ & 1727 & 2164 & 90317,23 & 301,06 \\
\hline$\$ 320000$ & 11,64 & VIII & $\begin{array}{l}\text { Sta } \\
\text { Victoria }\end{array}$ & $8,12 \%$ & $\$ 809621$ & 1253 & 1681 & 70128,02 & 233,76 \\
\hline$\$ 380000$ & 13,82 & IX & $\begin{array}{l}\text { Santa } \\
\text { Isabel }\end{array}$ & $6,46 \%$ & $\$ 1122300$ & 1044 & 1984 & 82801,67 & 276,01 \\
\hline$\$ 380000$ & 13,82 & IX & $\begin{array}{l}\text { Santa } \\
\text { Isabel }\end{array}$ & $9,87 \%$ & $\$ 791228$ & 1959 & 2017 & 84170,52 & 280,57 \\
\hline$\$ 350000$ & 12,73 & VIII & $\begin{array}{l}\text { Santa } \\
\text { Isabel }\end{array}$ & $9,31 \%$ & $\$ 772835$ & 1682 & 1852 & 77263,34 & 257,54 \\
\hline
\end{tabular}




\begin{tabular}{|c|c|c|c|c|c|c|c|c|c|}
\hline $\begin{array}{l}\text { Precio de } \\
\text { Arriendo } \\
(\text { CLP) }\end{array}$ & $\begin{array}{l}\text { Precio de } \\
\text { Arriendo } \\
\text { (UF) }\end{array}$ & $\begin{array}{l}\text { Decil } \\
\text { que } \\
\text { puede } \\
\text { pagar }\end{array}$ & Ubicación & Yield & $\begin{array}{l}\text { Ingreso } \\
\text { para } \\
\text { Acceder a } \\
\text { Compra }\end{array}$ & $\begin{array}{l}\text { Utilidades } \\
\text { (UF) }\end{array}$ & VAN & $\begin{array}{l}\text { Plusavalía } \\
\text { a } 25 \text { años } \\
\text { (USD) }\end{array}$ & $\begin{array}{l}\text { Plusvalía } \\
\text { Anual } \\
\text { (USD) }\end{array}$ \\
\hline$\$ 370000$ & 13,45 & IX & Jofré & $7,65 \%$ & $\$ 969179$ & 1358 & 1944 & 81107,02 & 270,36 \\
\hline$\$ 380000$ & 13,82 & IX & Tarapacá & $11,84 \%$ & $\$ 662477$ & 2315 & 2030 & 84702,85 & 282,34 \\
\hline$\$ 470000$ & 17,09 & IX & $\begin{array}{l}\text { Sta } \\
\text { Victoria }\end{array}$ & $8,99 \%$ & $\$ 1022978$ & 2300 & 2496 & 104162,00 & 347,21 \\
\hline
\end{tabular}

Cuadro 3. Estudio de rentabilidad y precios de arriendo en el sector Santa Isabel en Santiago Centro. Selección de 31 casos representativos de un total de 1496 casos (21 de marzo de 2019). Fuente: Elaboración propia en base a levantamiento desde Toc-Toc, Portal Inmobiliario y Servicio de Impuestos Internos.
El factor Yield indica la rentabilidad de invertir en cada unidad observada. Para tener una referencia, el Yield de negocios similares a comprar-para-arrendar en la ciudad de Sídney se considera rentable cuando se ubica entre el $6 \%$ y $8 \%$ (Waltl, 2018). Además se calculó la rentabilidad medida en Valor Actual Neto (VAN) con una tasa de descuento de $6 \%$ (base + riesgo) y asumiendo una plusvalía promedio de $8 \%$, a partir del sector de estudio estimada con el modelo que utiliza proyecciones de datos de los últimos 5 años (Toc-Toc, 2019). El cálculo del VAN considera un escenario en el cual se compra la vivienda sobre la base de crédito hipotecario, en condiciones de mercado actual: $3,81 \%$ para la tasa de interés bancario + inflación anual para un periodo de 25 años de plazo para la liquidación total de la deuda, alquilando esta propiedad en precios de mercado publicados en los sitios antes mencionados considerando las variaciones futuras del IPC proyectado mediante la UF.

Dado que el Yield en los 31 casos evaluados es mayor al 8\%, se entiende que comprar-para-arrendar es un muy buen negocio. Ejemplo, un fondo mutuo en Chile renta como máximo en un $5 \%$, que es una de las alternativas para colocar estos montos de inversiones en instrumentos financieros con rentabilidad probada. Este modelo es una excelente iniciativa de inversión considerando el déficit estructural de oferta de vivienda en Chile (Rodríguez y Sugranyes, 2005). Al comparar qué tan rentables son las diferentes tipologías de vivienda estudiadas, se identifica que mientras menos recintos tiene la unidad de vivienda ( 1 dormitorio y 1 baño) mayor es su rentabilidad promedio $(9,75 \%$ de Yield). En el total de la muestra seleccionada se obtiene una rentabilidad promedio de $9,13 \%$ y con una superficie promedio de 43 $\mathrm{m}^{2}$, mientras que los más rentables ofrecen una superficie de $34 \mathrm{~m}^{2}$.

Uno de los instrumentos más utilizados para determinar la plusvalía de una inversión es el Valor Actual Neto. En este caso todos los departamentos de la muestra tienen un VAN superior a 0, por lo tanto, son todos rentables, alcanzando un promedio de $92,14 \%$ sobre el valor de compra inicial. Es decir, por comprar-para-arrendar se puede generar una máquina de inversionistificación eficiente que, en este caso, se presenta como una segura inversión en un capital de renta fija a largo plazo. Esto implica que la hipótesis de la vaca lechera se puede aplicar en este barrio. Es decir, a partir de una compra inicial de un departamento para rentar desde su uso se puede iniciar un proceso de expansión de cartera de viviendas para la renta como actividad económica. La particularidad de esta actividad económica es que en Chile no paga impuestos a la renta equivalentes al margen de rentabilidad aquí observados. Por lo tanto se constituye como una excelente alternativa de suplemento de bienestar económico a futuro, en una nación que ha privatizado la seguridad social (Solimano, 2012). No obstante, como se indica en la tabla, en el caso observado, en la actualidad es solo accesi- 
ble para los deciles de ingreso entre el VIII y el X, producto del aumento del precio del arriendo y los precarizados presupuestos familiares que en Chile, además de vivienda, generalmente pagan salud y educación (Vergara-Perucich, 2018).

\section{Conclusiones}

Retomando la teoría de Kath Hulse y Margaret Reynolds (2018), la investification es un proceso en el que un inversor adquiere propiedades en barrios de menores ingresos, aumentando el precio de dichas propiedades y presionando los alquileres en el sector con nuevos precios que son pagados por segmentos socioeconómicos de menores ingresos en un modelo rentista. Según los datos aquí presentados, en Chile existe un proceso activo de inversionistificación porque deciles VIII, IX y X capitalizan la compra de viviendas en sectores de ingresos medios, aumentando su precio de arriendo y la consecuente mercantilización de la vivienda en una lógica rentista.

Debido a la fragilidad de la seguridad social en Chile, este fenómeno podría no tener que ver directamente con la avaricia sino con la búsqueda de un refugio económico para asegurar un bienestar a futuro con los espacios que entrega el modelo neoliberal. Aun así, esta rentabilidad, al no pagar impuestos, deja de contribuir a mejorar el bienestar de la población y, también en algunos casos, se puede decir que el factor avaricia podría ser determinante en ampliar considerablemente el stock de vivienda en un solo hogar en un modelo de acumulación por desposesión (Harvey, 2006) dentro del marco de la inversionistificación urbana (Hulse y Reynolds, 2018). Para Robert Hardaway (2011), en el estudio de crisis inmobiliarias el factor avaricia hace referencia a una oportunidad en el mercado que es sobreexplotada por consumidores que evalúan la rentabilidad a corto plazo sin tomar resguardos de sus riesgos a largo plazo. Esto se aplica tanto para individuos como para instituciones financieras (ob. cit.). Una de las preguntas pendientes de este estudio es cómo identificar si un propietario que arrienda sus viviendas lo hace por rentismo puro o a causa de la fragilidad del bienestar social que ofrece el estado neoliberal de Chile. Independiente de lo anterior, constatamos la inexistencia de instrumentos de política pública que permitan controlar, regular u organizar este proceso económico, con miras a disminuir su impacto en el precio de la vivienda y en la posibilidad de desarrollar un sistema urbano más equitativo.

El aumento significativo del esfuerzo presupuestario que hacen los hogares para pagar el arriendo cuenta con un correlato hacia otros bienes que estos hogares deben realizar en Chile que no son gratuitos: agua, energía, calefacción, transporte, alimentación, educación, salud y ocio. De continuar este aumento generaría un efecto de sustitución en alguno de estos servicios de bienestar social, precarizando aún más el presente de los hogares en desmedro del rentismo de otras actividades sociales que también operan bajo los marcos del modelo neoliberal monetarista chileno. La vivienda es un bien de consumo básico y un derecho humano que se encuentra amenazado por una mercantilización de este bien con bajos niveles de regulación, algo que agrede la seguridad social. La Constitución chilena establece en su artículo 18 el derecho a la seguridad social. Por esta razón, proponemos crear una superintendencia de vivienda, que empodere el actual Observatorio de Vivienda del Ministerio de Vivienda y Urbanismo. Esta superintenden- 
cia debería vigilar los procesos de asignación de unidades de vivienda de acuerdo a los déficits estructurales en las comunas de Chile, transparentando los datos del mercado de vivienda en ventas y arriendo. Sin duda, estos datos son de alto interés público y no debiesen dificultar su acceso para el análisis científico de las dinámicas de la vivienda en la nación. En lo concreto, tanto datos del Ministerio de Vivienda y Urbanismo, como del Servicio de Impuestos Internos, Notarías, portales de mercado inmobiliario y de los Conservadores de Bienes Raíces debieran de transparentar sus datos y ponerlos a su libre disposición para consulta, análisis y seguimiento. La experiencia internacional dice que la oscuridad en procesos de mercado de vivienda pueden contribuir a la producción de crisis económicas. También esta superintendencia tendría la misión de mediar entre ciudadanos y empresas a la hora de transar viviendas o bien de prestar servicios inmobiliarios. Desde una aplicación práctica de política pública, sugerimos que para casos de inversionistificación, una agencia especializada (la misma superintendencia) debiera de tener las atribuciones para revisar caso a caso los procesos en los que se comercializa el que una vivienda se vende o arrienda, y así determinar si la compra de vivienda obedece a un proceso de explotación especulativa o bien para explotar su valor de uso, privilegiando este último. Ejemplo, un individuo puede comprar tantos departamentos como familiares dependientes tiene, con el objeto de capitalizar un buen momento económico personal y asegurar el techo para sus hijos, padres, pareja o familiares en primer o segundo grado. Si se excede de este número, se le aplica una tarifa de impuesto a la renta por las propiedades o bien, se le exige que se constituya como empresa que debe tributar por dichas actividades. De esta manera, el proceso asume la fragilidad financiera y de seguridad social en Chile donde la propiedad se convierte en un activo paliativo de eventuales vulnerabilidades futuras, a la vez que desincentiva el rentismo con propiedades.

$\mathrm{El}$ arriendo de vivienda ha tenido poco estudio reciente y actualizado en el contexto de las ciencias sociales en Chile. No obstante, a partir de los datos que aquí se han presentado, pareciera ser del todo significativo problematizar su impacto en los presupuestos familiares y en el bienestar general de la sociedad con especial énfasis en la clase media. Esta expresión de un modelo rentista no cuenta con los instrumentos de regulación ni de seguimiento por organismos públicos y autoridades, lo cual lo coloca en un espacio de privilegio aún mayor para quienes saben cómo invertir a partir del modelo de inversionistificación como un sistema de vaca lechera que reproduce la captación de recursos extraídos directamente de los bolsillos de la clase media chilena. 


\section{Referencias bibliográficas}

Boano, Camillo y Vergara-Perucich, Francisco (2017). Neoliberalism and Urban Development in Latin America: The Case of Santiago. Oxon-New York: Routledge.

Brenner, Neil, Marcuse, Peter y Mayer, Margit (2012). Cities for people, not ofr profit. New York-Oxon: Routledge.

Cociña, Camila (2016). Habitar desigualdades: Politicas urbanas y el despliegue de la vida en Bajos de Mena. Serie Documentos de Trabajo PNUD- Desigualdad, 2016/05, 1-21.

Duffie, Darrell y Kan, Rui (1996). A yield-factor model of interest rates. Mathematical Finance, 6(4), 379406. DOI: 10.1111/j.1467-9965.1996.tb00123.x

Durand, Cédric (2017). Fictitious Capital. How finance is appropriating our future. London-New York: Verso Books.

Flores, Pablo (2017). Migración y vivienda: apuntes para la política pública. Revista CIS, 7(22), 7-9. Recuperado el 12 de junio de 2019, de: http:/ / revistacis.techo.org/index.php/Journal/article/ view/19/29.

Gasic, Ivo (2018). Inversiones e intermediaciones financieras en el mercado del suelo urbano. Principales hallazgos a partir del estudio de transacciones de terrenos en Santiago de Chile, 2010-2015. Eure, 44(133), 29-50. DOI: 10.4067/s025071612018000300029

Gilbert, Alan (2013). How to help, and how not to help, the poor in the megacities of the South. City, 17(5), 628-635. DOI: $10.1080 / 13604813.2013 .827838$

Glaeser, Edward L. y Meyer, John Robert (2002). Chile. Political Economy of Urban Development. Cambridge, MA: Harvard University Press.

Graham, Stephen (2016). Vertical : the city from satellites to bunkers / Stephen Graham. The city from satellites to bunkers. London - New York: Verso Books.

Hanafizadeh, Payam y Latif, Vahideh (2011). Robust net present value. Mathematical and Computer Modelling, 54(1-2), 233-242. DOI: 10.1016/j. mcm.2011.02.005

Hardaway, Robert M. (2011). The Great American Housing Bubble: The Road to Collapse. Santa Barbara, CA: ABC-CLIO.

Harvey, David (2006). Neo-liberalism as creative destruction. Geografiska Annaler, Series B: Human Geography, 88(2), 145-158. DOI: 10.1111/j.04353684.2006.00211.x

Harvey, David (2015). Seventeen Contradictions and the End of Capitalism. Londres: Oxford University Press.

Hidalgo Dattwyler, Rodrigo (2014). La Ciudad Neoliberal: estímulos de reflexión crítica. En Hidalgo
Dattwyler, Rodrigo y Janoschka, Michael (Eds.), La Ciudad Neoliberal: Gentrificación y Exclusión En Santiago de Chile, Buenos Aires, Ciudad de México y Madrid (pp. 7-32). Santiago de Chile: Pontificia Universidad Católica de Chile.

Hulse, Kath y Reynolds, Margaret (2018). Investification: Financialisation of housing markets and persistence of suburban socio-economic disadvantage. Urban Studies, 55(8), 1655-1671. DOI: $10.1177 / 0042098017734995$.

Landon, Paulette (ed.) (2017). Encuesta Chile Dice. Capítulo 2. Santiago de Chile: Universidad Alberto Hurtado. Recuperado el 29 de junio de 2019 de: http://chiledice.uahurtado.cl/pdf/capitulo_2.pdf.

Instituto Nacional de Estadísticas (2018). Segunda entrega de resultados definitivos CENSO 2017. Santiago de Chile: Instituto Nacional de Estadísticas de Chile. Recuperado el 15 de junio de 2019, de: http:/ / www.censo2017.cl/wp-content/uploads/2018/05/ presentacion_de_la_segunda_entrega_de_resultados_censo2017.pdf.

Inzulza-Contardo, Jorge (2016). Contemporary Latin American gentrification? Young urban professionals discovering historic neighbourhoods. Urban Geography, 37(8), 1195-1214. DOI: 10.1080/02723638.2016.1147754

Inzulza, Jorge y Galleguillos, Ximena (2014). Latino gentrificación y polarización: Transformaciones socioespaciales en barrios pericentrales y periféricos de Santiago, Chile. Revista de Geografia Norte Grande, 58(5-6), 135-159. DOI: 10.4067/S071834022014000200008

Kolb, R. W. (2018). Net Present Value. In The SAGE Encyclopedia of Business Ethics and Society [versión electrónica]. DOI: 10.4135/9781483381503.n841

Leal, Jesús (2012). Residential segregation. En Smith, Susan J. (Ed.), International Encyclopedia of Housing and Home (Vol. 6, pp. 94-99). Cambridge: Elsevier. DOI: 10.1016/B978-0-08-047163-1.00566-X

Lees, Loretta, Slater, Tom y Wyly, Elvin (2008). Gentrification. Nueva York, Routledge. DOI: 10.4324/9780203940877

Lefebvre, Henri (1991). Critique of Everyday Life Volume 1. Geografiska Annaler. Series B, Human Geography (Vol. 77). London - New York: Verso. DOI: $10.2307 / 490375$

López-Morales, Ernesto (2016). Gentrification in Santiago, Chile: a property-led process of dispossession and exclusion. Urban Geography, 37(8), 1109-1131. 10.1080/02723638.2016.1149311

López-Morales, Ernesto, Gasic Klet, Ivo y Meza Corvalán, Daniel (2014). Captura desigual de renta y desplazamiento exclusionario. Indicadores generales 
del proceso de gentrificación en Santiago de Chile , 2000-2012. Cadernos Metrópole, 16(32), 565-586. DOI: 10.1590/2236-9996.2014-3212

López-Morales, Ernesto, Arriagada-Luco, Camilo y Gasic-Klet, Ivo y Meza-Corvalan, Daniel (2015). Efectos de la renovacion urbana sobre la calidad de vida y perspectivas de relocalizacion residencial de habitantes centrales y pericentrales del Area Metropolitana del Gran Santiago. Eure, 41(124), 45-67. DOI: $10.4067 /$ S0250-71612015000400003

López-Morales, Ernesto y Herrera, Nicolás (2018, 25 de julio). Arriendos por las nubes: efecto de la creciente concentración de la propiedad. CIPER CHILE. Recuperado el 10 de enero de 2019, de: https:/ /ciperchile.cl/2018/07/25/arriendos-porlas-nubes-efecto-de-la-creciente-concentracion-dela-propiedad/.

MIDESO, Ministerio de Desarrollo Social (2017). Informe de Desarrollo Social 2017. Santiago de Chile: Gobierno de Chile. Recuperado el 15 de junio de 2019, de: http:/ /www.ministeriodesarrollosocial. gob.cl/pdf/upload/IDS2017.pdf.

MIDESO (2018). CASEN, Encuesta de Caracterización Socioeconómica Nacional. Santiago de Chile: Gobierno de Chile. Recuperado el 15 de junio de 2019, de: http:/ /observatorio.ministeriodesarrollosocial.gob. cl/casen/casen_obj.php .

Morales Soler, Eva, Alonso Mallén, Rubén y Moreno Cruz, Esperanza (2017). La vivienda como proceso. Estrategias de flexibilidad. Hábitat y Sociedad, 4, 3354. DOI: 10.12795/habitatysociedad.2012.i4.03

Navarrete, Pablo y Navarrete, Nicolás (2016, 10 de julio). Moving "away" from Opportunities?: Homeownership and Employment. CAF, Working Paper $\mathrm{N}^{\circ}$ 2016/07. Recuperado el 12 de junio de 2019, de: http://scioteca.caf.com/handle/123456789/956.

OECD (2013). OECD Urban Policy Reviews, Chile 2013. Paris: OECD Publishing. DOI: 10.1787/9789264191808-en

Olivera, Patricia E. (2014). Neoliberalismo en la ciudad de México: Polarización y gentrificación. En Hidalgo Dattwyler, Rodrigo y Janoschka, Michael (Eds.), La Ciudad Neoliberal: Gentrificación y Exclusión En Santiago de Chile, Buenos Aires, Ciudad de México y Madrid (pp. 151-177). Santiago de Chile: Pontificia Universidad Católica de Chile.

Pagano, Michael A. y Bowman, Ann O’M. (1997). Cityscapes and Capital. Baltimore: The John Hopkins University Press.

Rodríguez, Alfredo y Sugranyes, Ana (2005). Los con Techo. Un Desafí para la Política de Vivienda Social. Santiago de Chile: Ediciones Sur.

Rodríguez, Alfredo y Sugranyes, Ana (2017). La Nueva Agenda Urbana: pensamiento mágico. Hábitat y Sociedad, 10, 165-180. DOI: 10.12795/HabitatySociedad.2017.i10.10

Rolnik, Raquel (2017). La guerra de los lugares. La colo- nización de tierra y la vivienda en la era de las finanzas. Santiago de Chile: LOM Ediciones.

Ruiz-Tagle, Javier y López M., Ernesto (2014). El estudio de la segregación residencial en Santiago de Chile: revisión crítica de algunos problemas metodológicos y conceptuales. EURE, 40(119), 25-48. DOI: $10.4067 / \mathrm{S} 0250-71612014000100002$

Sabatini, Francisco, Rasse, Alejandra, Cáceres, Gonzalo, Robles, María Sarella y Trebilcock, María Paz (2017). Promotores inmobiliarios, gentrificación y segregación residencial en Santiago de Chile. Revista Mexicana de Sociologia, 79(2), 229260. Recuperado el 30 de junio de 2019, de: http:/ / www.scielo.org.mx/scielo.php?pid=S018825032017000200229\&script=sci_abstract.

Solimano, Andrés (2012). Chile and the neoliberal trap: the post-Pinochet era. Cambridge: Cambridge University Press.

Swain, Dan (2012). Alienation, an introduction to Marx's theory. London: Bookmarks Publications.

Toc-Toc (2019). Arriendo y Venta de propiedades en Santiago Centro [Fuente de Datos]. Recuperado el 21 de Marzo de 2019, de: www.toctoc.com.

Tsatsaronis, Kostas, y Zhu, Haibin (2004). What drives housing price dynamics: cross-country evidence. BIS Quarterly Review, March, 65-78. Recuperado el 12 de junio de 2019, de: https://www.bis.org/ publ/qtrpdf/r_qt0403f.pdf.

Vargas, Miguel (2016). Tacit collusion in housing markets: the case of Santiago, Chile. Applied Economics, 48(54), 5257-5275. DOI: $10.1080 / 00036846.2016 .1176111$.

Vergara-Perucich, José Francisco (2017). Expoliación de la vivienda como activo financiero de renta fija en Antofagasta. Departamento de Economía- UCN (Chile), Working Progress n. ${ }^{\circ}$ 73. DOI: 10.13140/ RG.2.2.21604.83840

Vergara Perucich, José Francisco (2018). Estudio exploratorio sobre asequibilidad económica a la vida cotidiana en principales ciudades de Chile. Serie de Documentos de Trabajo en Economía-UCN (Chile), Working Paper n. ${ }^{\circ}$ WP2018-01. DOI: 10.13140/ RG.2.2.32344.55046

Vergara Vidal, Jorge Eduardo (2017). Verticalización. La edificación en altura en la Región Metropolitana de Santiago (1990-2014). Revista INVI, 32(90), 9-49. Reucperado el 30 de junio de 2018, de: https://scielo.conicyt.cl/pdf/invi/v32n90/07188358-invi-32-90-00009.pdf.

Waltl, Sofie R. (2018). Estimating quantile-specific rental yields for residential housing in Sydney. Regional Science and Urban Economics, 68(February 2017), 204-225. DOI: 10.1016/j.regsciurbeco.2017.10.011

Yaluff Portilla, Felipe (2016). Los secretos de la inversión inmobiliaria. Santiago de Chile: Editorial Un Nuevo Día. 
Vergara-Perucich, José-Francisco y Aguirre Nuñez, Carlos (2019). Inversionistificación en América Latina: problematización del mercado de arriendo para el caso chileno. Hábitat y Sociedad, 12, 11-27.

<http:/ /dx.doi.org/10.12795/HabitatySociedad.2019.i12.02> 


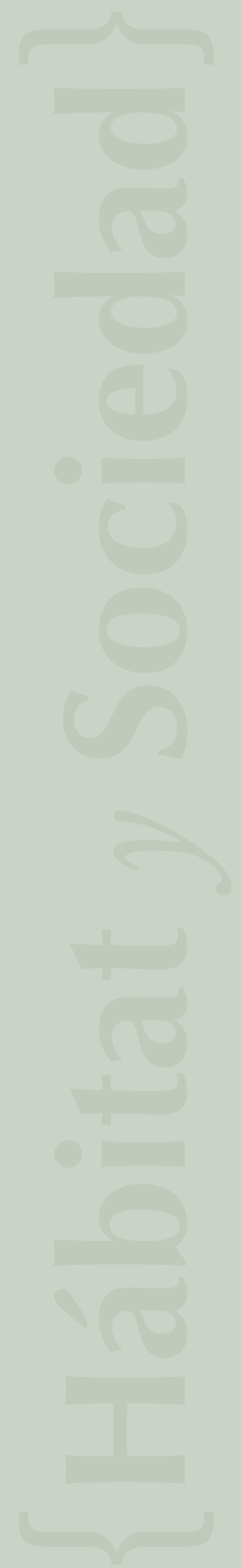

